developed for gastroenterology and surgical trainees and consists of a series of one day training events spread over several months to supplement departmental colonoscopy training and uses a variety of classroom based and simulation training. To facilitate the teaching of NTS we developed a video of a simulated case to form the basis of discussion.

Methods A video was made of a simulated case that could be used to highlight NTS. As the attached video demonstrates the video highlighted a case where the use of non-technical skills could be optimised. This was used as a starting point for discussion with delegates.

Pre and post course confidence ratings were collected on a variety of areas including human factors, mean ratings were calculated and mann-whitney $\mathrm{U}$ test applied.

Results Eight candidates completed the course, four surgical and four gastroenterology trainees. Six had already the JAG basic skills course. Confidence rating in human factors improved from a mean of 5.9 (out of 10) to 8.12 (p 0.03)

Conclusions Emphasis on non-technical skills training is imperative to improve and ensure patient safety and minimise adverse events. The teaching of non-technical skills may not always be explicit within working departments. Whilst simulation can be a useful teaching tool for NTS it can be time consuming This video demonstrates a resource-low method by which this could be taught as part of a wider endoscopy course. Whilst we only measured self ratings rather than assessing performance trainees confidence rating in human factors significantly improved suggesting this is a useful teaching tool.

\section{PTH-070 SEVERE MIDGUT BLEEDING FROM JEJUNAL DIVERTICULA TREATED BY DOUBLE-BALLOON ENTEROSCOPY}

${ }^{1}$ Alberto Murino, ${ }^{1}$ Nikolaos Lazaridis*, 'Nikolaos Koukias, ${ }^{1}$ Andrea Telese, 'Deborah Costa, ${ }^{1}$ Claudia Coppo, ${ }^{1,2}$ Yoshikazu Hayashi, 'Edward I Despott. 'The Royal Free Unit for Endoscopy, The Royal Free Hospital And University College London (UCL) Institute For Liver And Digestive Health, London, UK; ${ }^{2}$ Division of Gastroenterology, Department of Medicine, Jichi Medical University, Shimotsuke, Japan

\subsection{6/gutjnl-2019-BSGAbstracts.95}

Introduction Small bowel diverticula are a rare cause of gastrointestinal (GI) bleeding. Their pathogenesis is still unclear and can be found in up to $1-2 \%$ of the general population. Although these lesions are usually asymptomatic, mid-gut bleeding from diverticula in the jejunum or ileum could lead to a life-threatening situation, warranting emergency invasive therapy and often abdominal surgery.

Aims and Methods Our aim was to demonstrate the usefulness of the double-balloon enteroscopy (DBE) in the setting of an acute, severe small bowel diverticular bleed. A 79-year-old woman with hypertension and type II diabetes mellitus was referred to our institution with melaena and severe anaemia requiring urgent, repeat blood transfusions. Bidirectional conventional endoscopy did not reveal the cause of bleeding. Small bowel capsule endoscopy (SBCE) showed multiple diverticula within the jejunum and ileum. Emergency computed tomography (CT) mesenteric angiography demonstrated a faint 'blush' at one of the jejunal lesions.

Results Once the patient $\mathrm{w}$ as haemodynamically stable, emergency anterograde DBE was performed under general anaesthesia (GA) in our main operating theatres. The enteroscope was inserted into the jejunum, approximately 1.5 meters post- pylorus. The culprit cause of the bleeding was identified within a large $(5 \mathrm{~cm}$ orifice $)$ diverticulum, where a large, adherent, pulsating blood clot was seen. In the first instance, peri-lesion, quadrantic injection of a total of $20 \mathrm{mls}$ of adrenaline solution ( 1 in 10,000 dilution) was performed. The clot was then cautiously removed with a long endoclip to reveal the actively bleeding vessel which was then promptly clipped. A total of 3 clips were placed for effective haemostasis and a submucosal tattoo was placed adjacent to the bleeding point for future reference. The patient remained stable after the procedure and did not require any further blood transfusion.

Conclusion DBE facilitated endotherapy is a precise, safe and minimally invasive approach to the effective management of severe bleeding caused by small bowel diverticula.

\section{PTH-071 COMBINED ENDOSCOPIC CLOSURE OF COLOVESICAL FISTULA}

Faisal Nawaz*, Hemant Ojha, Monzur Ahmed. Good Hope Hospital, Birmingham, UK

\subsection{6/gutjnl-2019-BSGAbstracts.96}

Introduction Colovesical fistulas (CVFs) occur as a complication of diverticulitis, cancer, or Crohn's disease. The sigmoid colon is the most frequently involved segment. The most common presenting symptoms are pneumaturia and dysuria, followed by faecaluria, abdominal pain and, rarely, haematuria. The diagnosis is usually made clinically but can be confirmed by cystoscopy, sigmoidoscopy, barium enema, CT scan, MRI, or virtual colonoscopy. The usual management for symptomatic patients is colonic resection (which can be performed laparoscopically) but this approach is associated with morbidity and mortality. Only a few cases of endoscopic management by clip closure, using through-the-scope (TTS) or over-the-scope (OTS) clips have been described in the literature. Here, we present a case of a CVF, which was managed with multiple TTS clips, thus avoiding surgical intervention.

Methods A frail 84 year old man had a background history of coronary artery bypass graft, aortic valve replacement (tissue valve), dialysis dependent chronic renal failure and sigmoid diverticular disease. He was investigated for pneumaturia and recurrent urinary tract infections 12 months ago and was diagnosed on CT scan as having CVF secondary to sigmoid diverticular disease. He developed faecaluria, He was referred by the colorectal surgeon for endotherapy as he was considered too frail for any surgical intervention. A combined simultaneous cystoscopy and flexible sigmoidoscopy (using a gastroscope) was undertaken under conscious sedation. The cystoscopy identified a fistula in the dome of the bladder (on left side). The sigmoid colon was carefully examined and revealed diverticular disease but no obvious fistula was visible. Hence, indigocarmine solution (16 mg/litre), warmed to body temperature, was instilled into the bladder via the cystoscope and the blue solution was eventually seen to emerge in the sigmoid colon through the fistula. This site was marked with a Resolution 360 clip (Boston Scientific). After this, 4 further clips were placed onto the fistula in order to close it. Finally, the site was marked with a tattoo, in case a repeat procedure was needed in the future.

Results The procedure took almost two hours and was successfully performed. The patient was discharged on the same day. 